\title{
A Deep Spectral Survey of Peculiar A-Type Stars
}

\author{
Omar M. Kurtanidze, Miriam G. Nikolashvili
Abastumani Astrophysical Observatory, 383762 Abastumani Republic of Georgia

\begin{abstract}
We are conducting a long-term deep spectral survey to investigate the distribution of Ap and Am stars in selected fields of the Milky Way and in open star clusters of different ages. The spectral survey is based on objective prism spectra obtained with an eight degree prism attached to the $70 / 98 \mathrm{~cm}$ meniscus telescope $\left(\mathrm{f} / 3,135 \AA \mathrm{mm}^{-1}\right.$ at $\mathrm{H} \delta$ ) at the Abastumani Observatory. Kodak IIIa-J emulsions hypersensitized by baking in nitrogen gas are used in combination with a $500 \AA$ FWHM filter centered on $\mathrm{H} \delta$. A limiting magnitude of $m_{B}=14$ was reached in an exposure time of $120 \mathrm{~m}$. Study of the objective prism plates is still in a very early stage. Observations of the central part of the same field taken with a CCD resulted in a limiting magnitude $m_{B}=16$. On the basis of spectral material obtained, a wide range of spectra may be classified in the MK system.
\end{abstract}

\section{Introduction}

The main sequence A-type stars occur in a region of the Hertzsprung-Russell (HR) diagram where atmospheric models based on the classical assumptions of hydrostatic and radiative equilibrium might be expected to achieve their greatest success in describing the observed spectra. In most parts of the HR diagram "normal" stars are in the majority. Among A-type stars, however, abnormalities are the rule, rather than the exception (Wolff 1983).

The Ap stars are characterized by unusually strong lines of one or more of the following elements: Si 4128/30; Cr 4111, 4171; Sr 4077, 4216. They are classically defined to have temperatures like those of normal stars with spectral types from late B to late A. The frequency of close binaries seems to be much lower among Ap stars than it is among A-type stars without detectable magnetic fields. The Ap stars rotate much more slowly on average than do normal stars of the same temperature.

The Am phenomenon is present in stars that have an apparent surface under abundance of the Fe group (Sr II 4077 is more pronounced). The majority of Am stars with spectral types later than A4 are members of spectroscopic binary systems (Abt 1961). The discovery of several Am stars in the Orion Ia association (Smith 1972) demonstrates that the Am mechanism becomes operative and stabilized within about one million years. The occurence of Ap and Am stars in open clusters was studied by Abt (1979). Around $25 \%$ of main sequence A-type stars are Am, and about $10 \%$ are Ap stars (Wolff 1983, Lance 1988). A 
catalogue of 6684 chemically peculiar A stars has been compiled by Renson et al. (1991).

\section{The Survey}

Objective prism plates for the survey were taken with an eight degree prism attached to the $70 / 98 \mathrm{~cm}$ meniscus telescope (f/3). Kodak IIIa-J emulsions hypersensitized by baking in nitrogen gas are exposed for 30,60 or 120 minutes. Each field is round, $4.2^{\circ}$ in diameter, and covers 14 square degrees of the sky at a scale of $98 \mathrm{arcsec} / \mathrm{mm}$. The reciprocal linear dispersion and spectral resolution of the spectra obtained are equal to $135 \AA \mathrm{mm}^{-1}$ at $\mathrm{H} \delta$ and $3 \AA$, respectively.

Thanks to the high detector quantum efficiency and resolution of the Kodak IIIa-J emulsion, the excellent seeing at Abastumani (especially in August, September and October), the good optical quality of the meniscus telescope (Kiladze 1959) and the dark sky $m_{B}=22.5$ (Abuladze et al. 1981), it was easy to reach $m_{B}=14$ and to identify $A m$ and Ap stars. The fields under study are located in the longitude range $l=100-150^{\circ}$ and $|b|<5^{\circ}$. They are listed in Table 1 and selected from McCuskey et al. (1971).

Table 1.

\begin{tabular}{rrccc}
\hline No. & $\begin{array}{r}\alpha(1950) \\
h m\end{array}$ & $\begin{array}{c}\delta(1950) \\
\circ\end{array}$ & $\begin{array}{c}l \\
\circ\end{array}$ & $b$ \\
\hline 1 & 2207 & 51.4 & 99.0 & -3.5 \\
2 & 2220 & 52.7 & 101.5 & -3.5 \\
3 & 2328 & 56.9 & 112.0 & -4.5 \\
4 & 2346 & 57.1 & 114.5 & -4.5 \\
5 & 0028 & 62.0 & 120.5 & -0.5 \\
6 & 0101 & 61.1 & 124.5 & -1.5 \\
7 & 0145 & 57.3 & 130.5 & -4.5 \\
8 & 0200 & 57.3 & 132.5 & -4.0 \\
9 & 0205 & 62.9 & 131.5 & +1.5 \\
10 & 0204 & 61.0 & 136.5 & +1.5 \\
11 & 0235 & 56.1 & 137.5 & -3.5 \\
12 & 0242 & 53.4 & 139.5 & -5.5 \\
13 & 0257 & 53.4 & 141.5 & -4.5 \\
\hline \hline
\end{tabular}

In addition, five fields $\left(1=104^{\circ}, b=5^{\circ} ; 114,3 ; 122,3 ; 145,2 ; 150,0\right)$ in which the surface density of B8-A0 main sequence stars is relatively low will be studied for comparison. The spectral material was taken for fields Nos. 5 and 6. About ten plates were obtained for every field and spectra were widened to $0.2-0.3 \mathrm{~mm}$.

All plates were exposed through a $500 \AA \mathrm{FWHM}$ filter centered on $\mathrm{H} \delta$. The filter reduces the sky background by a factor of at least three, and also helps to overcome overlapping of the spectra. A few plates have been taken through $200 \AA$ 
FWHM filters centered on $3950 \AA$ (HK) and on H $\delta$. Direct plates (IIIa-J+GG13) were also obtained for magnitude determination of identified stars.

\section{Preliminary Results}

Each of the spectral plates (IIIa-J $+500 \AA \mathrm{H} \delta$ ) were independently scanned by us using a binocular stereoscopic microscope fixed on a special table supplied with a micrometric screw. It has different magnifications. We worked at magnifications 6 or 8. During the identification of peculiar A-type stars we always used two spectral atlases (Houk et al. 1974; 1984) with a very high quality of reproductions, namely for the stars under study. The independent identifications were compared and the catalogue of suspected Ap and Am stars in fields Nos 5 and 6 was compiled. It contains more than five hundred peculiar stars.

Scanning of another pair of plates (IIIa-J $+200 \AA \mathrm{HK}$ and IIIa-J $+200 \AA \mathrm{H} \delta$ ) took considerably longer, but was more effective for detection of these stars due to the high signal-to-noise ratio obtained during the same exposure. In the last case only the central $1 \times 1$ square degrees were searched.

We now plan to obtain unwidened spectra of the same field through these three filters and compare results after the digitization of the plates. The limiting magnitudes will be increased by at least two magnitudes. The advantages of using narrow band filters may be realized only if the quality of the spectra obtained with them are sufficiently high. As is well known, longer exposure times degrade the image quality due to different sources.

A few pilot CCD observations in the 8-degree mode in field No. 5 and other ones resulted in a limiting magnitude of $m_{B}=16$ in an exposure time of $15 \mathrm{~min}$.

\section{Acknowledgements}

It is pleasure to thank the SOC for financial support which enabled me to attend this meeting and Dr. Sandra Harrison without whose active help it would have been impossible to attend.

\section{References}

Abuladze, O. P., Kurtanidze, O. M. \& Nikolashvili, M. G. 1981, unpublished Abt, A. H. 1961, Ap. J. Suppl., 6, 37

Abt, A. H. 1979, in Spectral Classification of Future, eds McCarthy M. F., Philip A. G. D., Coyne G. V., IAU Coll. 47, p.95

Houk, N., Irvine, N. \& Rosenbush D., 1974, An Atlas of Objective Prism Spectra, Univ. of Michigan

Houk, M. \& Newbery, M. V. 1984, A second Atlas of Objective Prism Spectra, Univ. of Michigan

Lance, K. M. 1988, Ap. J. Suppl., 68, 463

McCuskey, S. W. \& Houk, N. 1971, Astr. J., 76, 1117

Kiladze, R. I. 1959, Bull. Abastumani Astrop. Obs., 24, 35 
Renson, P., Gerbali, M. \& Catalani, A. 1991, Astr. Ap. Suppl., 89, 429 Smith, M. A. 1972, Ap. J., 175, 765

Wolf, S. 1983, The A Stars: Problems and Perspectives, NASA SP-463 